\title{
PRODUCT LIFE CYCLE DESIGN USING THE DFE WORKBENCH
}

\author{
Elena Man ${ }^{1}$, Juan Enrique Díez ${ }^{2}$, Camelia Chira ${ }^{3}$, Thomas Roche ${ }^{4}$ \\ National University of Ireland, Galway ; Galway Mayo Institute of Technology, Ireland ${ }^{2,3,4}$ \\ elena.man@nuigalway.ie, juanen10@teleline.es,camelia.chira@nuigalway.ie \\ thomas.roche@nuigalway.ie
}

\begin{abstract}
This paper proposes a new design framework for the development of Environmental Superior Products (ESPS) and based on this framework a new DFE methodology and tool called the DFE Workbench is presented. The DFE Workbench is a CAD integrated DFE tool developed to support the designer in the creation of ESPS. The tool has been extensively tested by multinational organisations from electronic and automotive sectors. This paper presents the testing of the latest versions of both DFE Workbench methodology and tool. Results and conclusions are drown and future work is proposed.
\end{abstract}

\section{PROBLEM STATEMENT}

With the emergence of new European policies, (e.g. Integrated Product Policy), emerging legislation (e.g. WEEE and EEE) and environmental standards (e.g. ISO 14000) manufacturers are being forced to move towards the development of ESPs. According to WEEE and EOLV manufacturers are obliged to take responsibility for waste management by implementing re-use, recycle and recovery policies for their products. Design of ESPs (through DFE practices) is an effective strategy for complying with environmental drivers. Design for the Environment (DFE) is defined by Fiksel to be, ".. the systematic consideration of design performance with respect to environmental health and safety objectives over the full product and process life cycle"(Fiksel 1996). It is clear from this definition that DFE approaches must take a more holistic view of the life cycle than traditional design methodologies. It is therefore necessary to consider the design process and associated models when developing approaches to design for environment tools and methodologies.

The design process can be defined as an information transformation process, transforming design requirements information through a number of phases into detailed product specifications. The information transformation process is effected through a series of recurrent problem solving cycles that are used effectively to evaluate diagnose and improve the design as the design evolves (Roche 2001). It is therefore essential that DFE tools and methodologies should support this process throughout all phases. The authors' research has identified a set of requirements for the development of the methodologies and tools to support the development of ESPs. These requirements include:

- Methodologies and tools must be integrated as early as possible in the design process, as well as being integrated throughout the design process. 
- All approaches must take a life cycle view for the development of ESPs as a high degree of coupling can occur between lifecycle product characteristics in the design process.

- Approaches must be developed to reduce the environmental impacts and to extend first life and post first life of products, e.g. design for reuse, remanufacture and recycling.

- $\mathrm{CAD}$ Integrated tools are likely to be a powerful medium through which to embed DFE tools to support the designer in the development of ESPs.

- It is important to develop web based DFE applications because of the ubiquity, standardised protocols and the need to support distributed teams in the design of environmentally superior products.

\section{ESP REQUIREMENTS}

Traditional models of the design process have focused on the development of tools to improve the performance of a part of the life cycle of the product, e.g. design for manufacture or design for assembly. The result is a proliferation of tools to aid the designer at individual life cycle stages (Ishii 1992, Molina 1995). As discussed in the previous section, new models must take a more holistic view, i.e. focus on the total life cycle system (see figure1), to include raw material extraction, manufacture use and end of life (Kimura 1997, Alting 1993, Alting 1997, Lee 1993, Warnecke 1996).

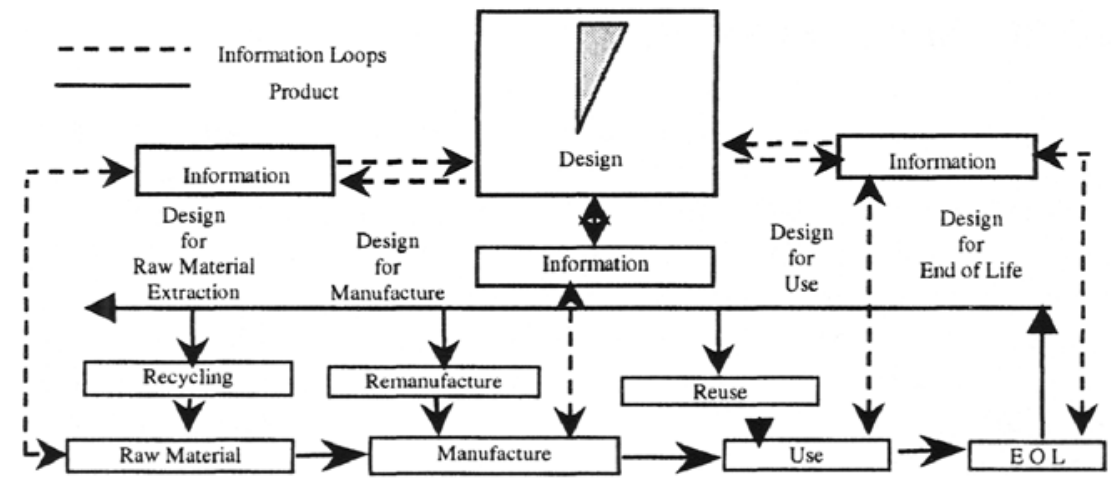

Figure 1. Design Information Loops (Roche 1999)

Four generic and interrelated strategies for the development of ESPs can be derived from the model as follows (Roche 1999):

- Select low impact materials and processes over all life cycle phases.

- Reduce life cycle resource consumption (Materials and Energy)

- Reduce life cycle waste streams (Materials and Energy).

- Resource sustainment by facilitating first life extension and post first life extension, i.e. reuse, remanufacture and recycling

In the model, shown in figure 1 life cycle information is acquired through a set of life cycle design information loops, i.e. design for raw material extraction, design for manufacture, design for use and design for end of life. The design process transforms this information into product design characteristics, which are subsequently embedded in the product. Therefore there is a need for a new design model to cater for the life cycle design information transformation loops and to support the development of new methodologies and tools to assist the designer in the creation of ESPs. 


\section{PAL FRAMEWORK}

Roche proposed that the life cycle design process could be represented by a tri-axial information transformation space, i.e. design phase, activity and information axes (see figure 2) (Roche 1999). The model represents the transformation of information through four generic stages of design (namely requirements definition, functional definition, general design and detailed design), i.e. the transformation of information from more abstract statements of requirements to more concrete details on the final design. The vertical axis is based on a synthesis of models (particularly prescriptive design process models) from the literature (Finger 1989, Cross 1994, Jones 1996, Pugh 1991, Hubka 1996, Waldron 1996, Evbuomwan 1996, Baya 1996, Pahl 1996). These phases are not discrete events within the design process, rather the designers engage in a set of decision-making cycles continuously improving the design at each level of abstraction. The problem solving cycle can be viewed as the instrument or mode of information transformation in each phase of the design process, hence a problem solving cycle is adopted to describe the activity axis of the design transformation space, i.e. the steps analyse, synthesise and evaluate (Hubka 1996, De Boer 1989, Cross 1994, Coyne 1990). The phase and activity axes define the boundaries of a design process plane. It is implicit in this plane that problem solving occurs explicitly at different levels of abstraction in the design process.

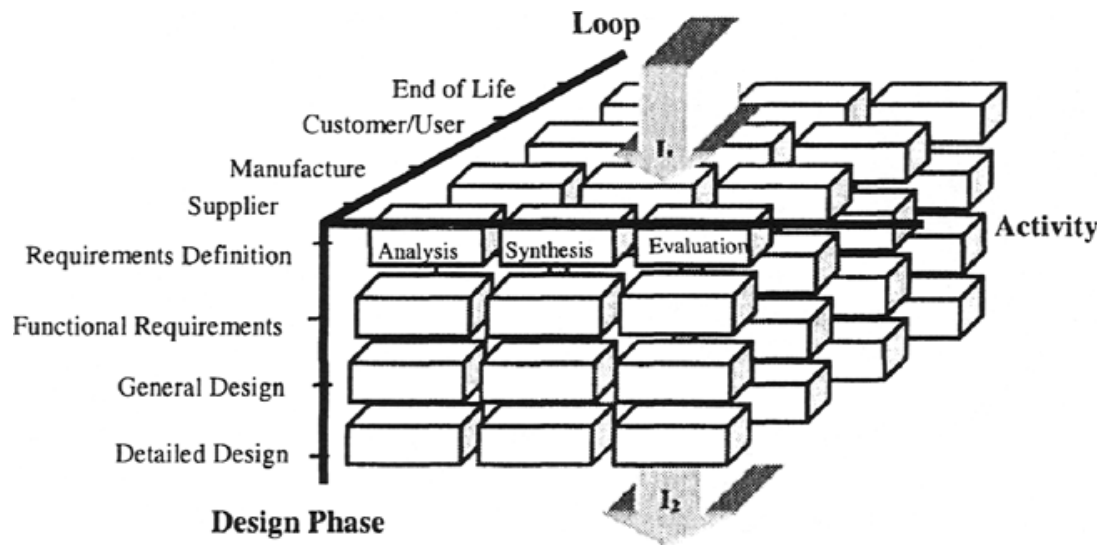

Figure 2. Tri-Axial Information Transformation Space for Life Cycle Design, (PAL)

Framework, Adopted for the Design of Extended Products (Roche 2001)

This affects the types of problem solving that can occur, and hence the types of tools and methodologies used.

As defined earlier ESPs require a life cycle design view. The design information loops (used to describe the third axis of the transformation space) described in figure 1 represent the source of information for each life cycle phase of the product and also as a focus for life cycle methodologies and tools. The activity and loop axes bound a life cycle problem-solving plane. This plane ensures the analysis, synthesis and evaluation of life cycle information throughout each phase of the design process. In summary the model in figure 2 , called the PAL framework, is proposed as a life cycle design framework to support the development of methods, methodologies and tools to aid life cycle design decisions. It is proposed to adopt this model for the development of information architectures, tools and methodologies to support the design of ESPs. 


\section{THE DFE WORKBENCH}

The development of the DFE Workbench methodology (based on PAL) is focused on the analysis, synthesis, evaluation and improvement of life cycle product General and Detailed design information. The DFE Workbench exists in two forms, firstly the manual methodology, which is largely based on using special charts and reference information in a structured manner to evaluate and improve an emergent design. The second is a CAD integrated software tool, which effectively automates processes, associated with the manual methodology, as well as providing added functionality such as a WEB based report generator. There has been much iteration within the development process where the authors' have developed, tested and improved previous versions of the tool from a simple desktop CAD integrated tool to a suite of integrated tools to be delivered across the enterprise. The tool has been extensively tested in both the electronic automotive sectors influencing the evolution of the tool. The DFE Workbench currently exists in three forms that can be configured to support three levels in the global enterprise: Desktop, Enterprise and

Global (see figure 3).

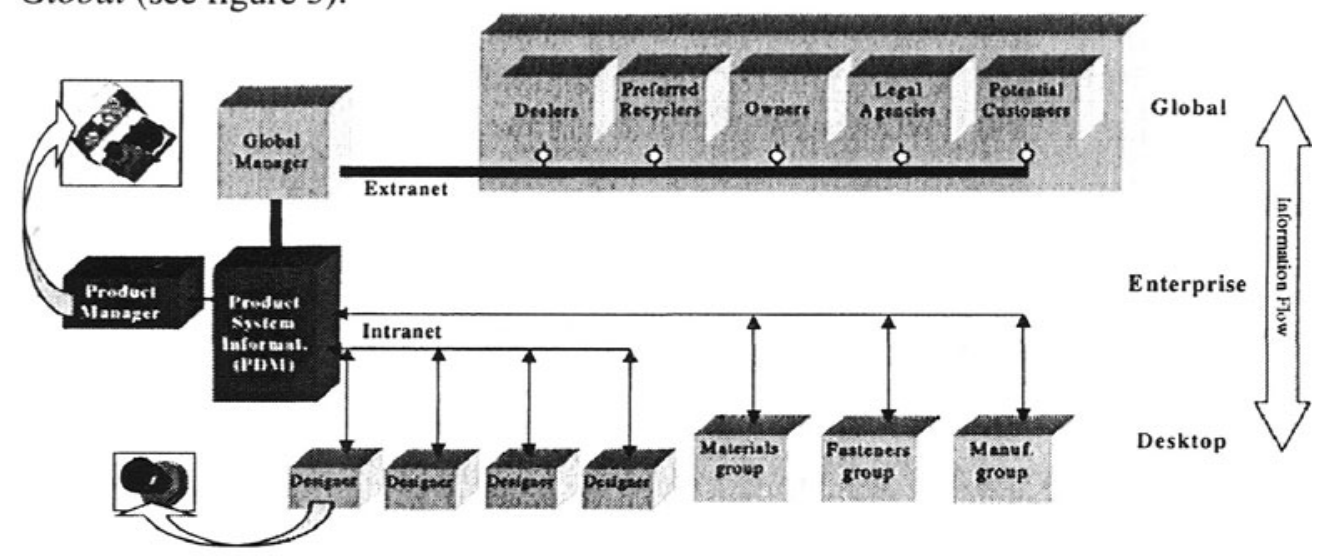

Figure 3. The DFE Workbench Structure

\subsection{DFE Workbench Desktop}

The DFE Workbench Desktop is a design for environment software tool integrated into a CAD environment developed to assist and advise the designer in the development of ESPs. The tool uses an intranet to connect to the main oracle databases and allows the designers to work concurrently on developing the product. The users have controlled access to the databases based on a username and a password. Currently the application has been ported to two CAD tools, namely Pro Engineer 2001 and Solid Works 2000. The appropriate data is automatically synthesised from the virtual prototype and evaluated using different DFE tools within the DFE Workbench. Each of the variables evaluated are prioritised and advice is given to the designer on alternative product or process characteristics that will enhance that variable. The designer optionally decides to accept the advice and makes the appropriate improvements. Data is then re-synthesised from the (new) model and the process begins again. The Desktop DFE Workbench consists of five modules: the Impact Assessment System (IAS), the Structure Assessment Method (SAM), the advisor agent, the knowledge agent and the dynamic report generator. 
The Impact Assessment System (IAS) is an abridged quantitative approach to LCA, performing synthesis, evaluation, prioritisation and improvement of environmental data. It automatically extracts the appropriate data from the virtual prototype. Impact data may be calculated for each part or for the entire assembly. Improvements can be made at the part or product system levels. There are several other metrics apart from the eco indicator calculated using the LCA that are evaluated, prioritised and improved, these include: Material Types and Variety, Material Intensity of Type/s (Mass), \% Recycled Material Content, \% Recyclable Material and \% Hazardous Material

The Structure Assessment Method (SAM) focuses on the structure of the emergent virtual prototype in an attempt to enhance structural characteristics of the product in the context of DFE. SAM is a complex methodology, which quantitatively measures and records data including such as material compatibility (taking into account fasteners), components serviceability, number and types of fasteners, number and types of tools required for disassembly or total standard disassembly time and part removal time. Coupling between all variables is managed and recorded by the DFE Workbench. For example if an additional fastener is added to the virtual prototype then the number and variety of fasteners and disassembly times and routes are recalculated for the product structure.

The Advisor Agent has two functions: firstly to prioritise variables generated by the IAS and SAM tools; secondly the advisor agent actively gives advice to the designer on alternative structural characteristics to enhance either the environmental impact or structural characterises of the emergent design.

The Knowledge Agent provides advice to the designer in a consultative mode. For example the designer can use the Knowledge Agent to find a material with specified mechanical and environmental properties. The designer can then use the selected material in the design process.

The Report Generator automatically generates reports on the product designed by the user. These reports are made available in two modes, i.e. as system reports that can be printed and viewed locally or as World Wide Web reports that can be made available via an extranet model to people who need product data. For example dismantlers may need to know the location of hazardous materials, the disassembly route and time for a specific product type. If the designer makes a change in the product structure in the design process then the data is automatically updated on the web server.

\subsection{DFE Workbench Enterprise}

The DFE Workbench Enterprise has been developed on the same principles as the DFE Workbench Desktop but it has been developed for the product system level and therefore gives a holistic view over the environmental and structural properties of the candidate design. The DFE Workbench Enterprise can work both outside and inside the $\mathrm{CAD}$ environment. It extracts relevant data and performs evaluation, prioritisation and improvement of the design solution. The user can focus on a subassembly structure and can drill down into component information when required. The application identifies the subassembly (or indeed the subassemblies) that has the highest environmental impacts or the undesired structural properties and will give advice on how to improve it. Functional departments within the enterprise can put 'standard' data into the DFE Workbench Databases and can synthesise data 
and generate reports as the design evolves. For example the materials department have access to the DFE Workbench Databases through a web-based interface that enables the addition of new materials and application advice for the designers. Additionally, they can view and generate reports and provide advice on an evolving design, e.g. product mass, hazardous material content, identify priority subassemblies and suggest alternatives.

\subsection{DFE Workbench Global}

The DFE Workbench Global has been developed as an Intranet/Internet tool that supports communications and reporting of the environmental and structural metrics that have been calculated, evaluated and improved using the DFE Workbench Desktop and Enterprise tools. The tool has been developed using Cold Fusion technology and works with the Oracle Databases used by the designer and the system/project engineer. It operates through a controlled environment and uses security protocols allowing various users to log in and view, upload and copy data that has been customised for their specific needs. The DFE Workbench Global may also be used via an intranet for interdepartmental reporting or information on specific issues like the preferred materials for specific components or preferred fasteners. The DFE Workbench Global has been developed as an extremely flexible application that supports a high degree of customisation.

\section{DFE WORKBENCH TESTS AND RESULTS}

The DFE Workbench methodology and the software tool have been extensively tested with the industrial partners from electronic and automotive industry. The tool has been developed through a number of iterations in an attempt to improve the functionality and the usability of the tool and also to ensure the compatibility of the tool with the needs of a corporate organisation. For the purpose of this paper this section describes the testing of the final versions of both manual methodology and software tool. The tests have been divided in two exercises as follows:

a) The analysis, synthesis, evaluation and improvement of an existing product using the manual method (a domestic smoke alarm).

b) The analysis, synthesis, evaluation and improvement of a virtual prototype of an automobile mirror in the design process, using the DFE Workbench software. A partner from the automotive industry provided the mirror.

The DFE Workbench methodology was tested using a protocol analysis technique involving he completion of a short designing exercise in the presence of a video camera. The subjects were provided with all the necessary materials to ensure the proper use of the manual methodology. At the end of the testing session each of the subjects were asked to fill in a set of questionnaires. The tests were carried out in the Research Lab at CIMRU, National University of Ireland Galway, and at the site of one of the industrial partners.

The objectives were twofold, i.e. to evaluate the functionality and usability of the DFE Workbench and to identify opportunities and obstacles for the customisation of the DFE Workbench to meet the requirements of a corporate organisation.

A summary of the conclusions on the DFE Workbench methodology is as follows: 
- The proper application of the methodology can result in the improvement of the design irrespective of the experience of the designer. Experience combined with the correct use of the methodology is an advantage.

- The manual method takes a long time to complete. It is tedious to calculate all of the variables, particularly when having to iterate through a number of solution variants and having to recalculate every time.

- Learning was observed whilst the subjects used the manual methodology. It is also expected that learning take place in the use of Software based workbench and probably at a faster pace. It was confirmed that this learning influenced decisions made later by the designers

- With proper training on the manual methodology a non-expert user can contribute to design issues, therefore the manual methodology can act as a training medium.

- The use of standardised criteria such as; standard times, labelling, and material compatibility's, is a very positive feature of the methodology particularly for benchmarking and design comparison.

- Because of the volume of calculations and the manipulation of interdependent relationships it is considered essential to develop a software application to support the methodology.

- The methodology would benefit from the inclusion of extra tools to support different types of decisions, e.g. a cost analysis tool.

- The strong and clear linkage between the global and local indices is identified as a very positive feature of the methodology.

- The prioritisation process was found to be very useful for the search and improvement activity.

- The inclusion of an advisor was seen to essential to the operation of the methodology.

The following conclusions resulted from the testing of the DFE Workbench software:

- There are very distinct advantages for integrating the DFE Workbench in a CAD environment, not least the automation of data synthesis activity, the availability of quantitative data directly from the model, the manipulation of this data, the management of data interrelationships, the accelerated learning that takes place as a result of active experimentation and the resulting improvement in a design before it is manufactured.

- The generation of results and the manipulation of data as well as the speed of improvements made were much faster and accurate.

- It is possible to make improvements on virtual models that may not be possible in real prototypes.

- It has been viewed that the tool would increase the productivity of the design process, would reduce time to market and would support the rapid achievement of design targets.

- The attendees felt that placement of such a tool right on the designers' desktops was a key advantage

- It has been identified the need for more accurate and customised databases to cover fasteners, materials and processes that are specific for the type of industry the tool is addressing e.g. in this case the automotive industry 


\section{ONGOING FURTHER DEVELOPMENT}

The DFE Workbench is undergoing continuous development at the authors' institution to meet the following goals:

- Construction of a Life Cycle Costing (LCC) module

- Addition of new evaluation tools apart from IAS and SAM

- Integration of a work-flow manager

- Development of new interfaces for various departments on the same organisation as for example materials department or fasteners department.

- Development of agents to handle the information management between design teams and rest of members in the organisation

- Development of a conceptual design tool to assist the decision making process associated with the development of various types of products as for example extended products, ESPs and one-use products.

\section{REFERENCES}

1. Alting L., Wenzel H., Hauschild M., "Environmental Assessment of Products", ChapmanHall, 1997.

2. Alting, L., "Life Cycle Design of Products: A New Opportunity for Manufacturing Enterprises", Concurrent Engineering Automation Tools and Techniques, Wiley Press, 1993, pp 1- 17.

3. Baya V., "Information Handling Behavior of Designers During Conceptual Design", Ph.D. Thesis, Stanford University, 1996.

4. Coyne R.D., Rosenman M.A., Radford M.A., Balachandran M., Gero J.S., "Knowledge based Design Systems", Addison Wesley, 1990

5. Cross N., "Engineering Design Methods", J. Wiley \& Sons, 1994

6. De Boer S. J., "Decision Methods and Techniques in Methodical Engineering Design", Ph.D. Thesis, University of Twente, 1989

7. Evbuomwan N.F.O., "A Survey of Design Philosophies, Models, Methods and Systems", In Proceedings of Instition of Mechanical Engineers, Vol 210, pp301-321,1996.

8. Fiksel J, "Design for environment creating eco-efficient products and processes", McGrawHill, 1996

9. Finger S., Dixon J.R.,"A Review of Research in Mechanical Engineering Design - Part 1Descriptive, Prescriptive and Computer Based Models of the Design Process", In Research in Engineering Design, pp 51 - 67, Springer Verlag, 1989.

10. Hubka V., Eder E., "Design Science", Springer, 1996

11. Ishii K., Hornberger L., " The Effective Use and Implementation of Computer Aids for Life Cycle Product Design", in Advances in Design Automation, Volume 1, ASME, 1992.

12. Jones J., "The Engineering Design Process", Wiley Press, 1996.

13. Kimura F., "Inverse Manufacturing: from Product to Services. Managing Enterprises-Stakeholders, Engineering, Logistics and Achievement". $1^{\text {st }}$ Int. Conf. Proceed. MEP, Ltd. London UK. 1997.

14. Lee D.E., "Issues in Product Life Cycle Engineering Analysis", in Advances in Design Automation, V 65-1, ASME, 1993.

15. Molina A., Al-Ashaab A.H., Ellis T.I., Young R., Bell R., "A review of Computer-Aided Simultaneous Engineering. Systems", Research in Eng. Design, V7, Springer-Verlag, 1995.

16. Pahl G., Beitz W., "Engineering a Systematic Approach", Springer, 1996

17. Pugh s., "Total Design", Addison Wesley, 1991

18. Roche, T. Man, E. Browne, J. "Development of a CAD integrated DFE Workbench tool", IEEE 2001 International Symposium on Electronics and the Environment, Denver, 2001

19. Roche, Thomas "The Development of a DFE Workbench", Ph.D. Thesis, September 1999.

20. Tomiyama, T., "The Technical Concept of Intelligent Manufacturing Systems (IMS)", University of Tokyo, internal document. 1994.

21. Waldron M.B., Waldron K.J., "Mechanical Design Theory and Methodology", SpringerVerlag, 1996

22. Warnecke G., "A Co-Operation Model of Product Development and Recycling", Proceedings of First International Seminar on Reuse, Eindhoven 1996. 